\title{
On two-weight inequalities for Hausdorff operators of special kind in Lebesgue spaces
}

\author{
Rovshan Bandaliyev*1,2 (D), Kamala Safarova $^{1}$ (iD \\ ${ }^{1}$ Institute of Mathematics and Mechanics of NAS of Azerbaijan, Baku, Azerbaijan \\ ${ }^{2}$ Peoples' Friendship University of Russia, Russia, Moscow
}

\begin{abstract}
In this paper, we establish necessary and sufficient conditions on monotone weight functions for the boundedness for Hausdorff operators of special kind in weighted Lebesgue spaces. In particular, we get similar results for important operators of harmonic analysis which are special cases of the Hausdorff operators. The weights are illustrated by examples at the end of the paper.
\end{abstract}

Mathematics Subject Classification (2020). 28C99, 46E30, 47G10

Keywords. Hausdorff operators of special kind, weighted Lebesgue spaces, monotone weight functions

\section{Introduction}

It is well known that Hausdorff operators have a deep root in the study of the Fourier analysis and it has a long history in the study of harmonic analysis. This integral operator is deeply rooted in the study of one-dimensional Fourier analysis and has become an essential part of modern harmonic analysis. In particular, it is closely related to the summability of the classical Fourier series [18].

Let $\phi$ be a locally integrable function on $\mathbb{R}_{+}$. Then the Hausdorff operator is defined by

$$
H_{\phi}(f)(x)=\int_{0}^{\infty} \frac{\phi\left(\frac{x}{y}\right)}{y} f(y) d y .
$$

Many important operators in analysis are special cases of the Hausdorff operator which can be obtained by taking suitable choice of $\phi$. Let us denote by $\chi_{E}$ the characteristic function of $E \subset \mathbb{R}_{+}$. For example,

1. if $\phi(t)=\frac{\chi_{(1, \infty)}(t)}{t}$, we get the Hardy operator

$$
H f(x)=\frac{1}{x} \int_{0}^{x} f(t) d t
$$

\footnotetext{
*Corresponding Author.

Email addresses: bandaliyevr@gmail.com (R.A. Bandaliev), kaama84@mail.ru (K.H. Safarova)

Received: 05.10.2020; Accepted: 09.04.2021
} 
2. if $\phi(t)=\chi_{(0,1)}(t)$, we have the adjoint Hardy operator

$$
H^{\star} f(x)=\int_{x}^{\infty} \frac{f(t)}{t} d t
$$

3. if $\phi(t)=\max \{1, t\}$, we get the Hardy-Littlewood-Pólya operator

$$
\operatorname{Pf}(t)=\frac{1}{x} \int_{0}^{x} f(t) d t+\int_{x}^{\infty} \frac{f(t)}{t} d t
$$

4. if $\phi(t)=\gamma(1-t)^{\gamma-1} \chi_{(0,1)}(t)$ with $\gamma>0$, we obtain the Cesàro operator

$$
C_{\gamma} f(x)=\gamma \int_{x}^{\infty} \frac{(t-x)^{\gamma-1}}{t^{\gamma}} f(t) d t
$$

In the last two decades various problems related to the Hausdorff operators attracted much attention in different applications. The Hausdorff operators has been extensively studied in recent years, particularly its boundedness in Lebesgue space. We also refer to $[1,2,7-10,12,15,16,20,21]$ for some recent works in this vein. Recently, two-weight inequalities in the framework of Hausdorff operators were studied in [4] and [19] (see, also [6]). We note that in [19] the obtained necessary condition for the boundedness of Hausdorff operator in weighted Lebesgue spaces differ from the sufficient condition and coincide for the Hardy and Bellman operators. Also, in [4] and [6] the obtained necessary condition for the boundedness of Hausdorff operator in weighted Lebesgue spaces differ from the sufficient condition. In the general case for the positive operators weighted integral inequalities were studied in [13]. We refer to [3,5,11,14,17,22] for more results on two-weight inequalities for fractional integral operators.

The main goal of the paper is to study the boundedness of Hausdorff operators of special kind in weighted Lebesgue spaces for monotone weight functions. We establish necessary and sufficient conditions on monotone weight functions for the boundedness of Hausdorff operators of special kind in weighted Lebesgue spaces.

The remainder of the paper is structured as follows. Section 2 contains some preliminaries along with the standard ingredients used in the proofs. Our principal assertions, concerning the continuity of Hausdorff operators of special kind in mentioned spaces are formulated and proven in Section 3. We establish necessary and sufficient conditions on monotone weight functions for the boundedness of Hausdorff operators of special kind in weighted Lebesgue spaces in Section 3. The weights are illustrated by examples in subsection 3.1 .

\section{Preliminaries}

We recall the definition of the weighted Lebesgue spaces.

Definition 2.1. Let $1 \leq p<\infty$ and let $p^{\prime}$ denote the conjugate exponent defined by $p^{\prime}=\frac{p}{p-1}$. Suppose $\omega$ is a weight function on $\mathbb{R}_{+}$, i.e. $\omega \in L_{1}^{\text {loc }}\left(\mathbb{R}_{+}\right)$and $\omega(x)>0$ almost everywhere. The weighted Lebesgue space $L_{p, \omega}\left(\mathbb{R}_{+}\right)$is the class of all Lebesgue measurable functions $f$ defined on $\mathbb{R}_{+}$such that

$$
\|f\|_{L_{p, \omega}\left(\mathbb{R}_{+}\right)}=\left(\int_{0}^{\infty}|f(x)|^{p} \omega(x) d x\right)^{\frac{1}{p}}<\infty .
$$

We note that for $\omega \equiv 1, L_{p, \omega}\left(\mathbb{R}_{+}\right)$means usual Lebesgue space $L_{p}\left(\mathbb{R}_{+}\right)$.

Throughout this paper, we denote by $C$ a positive constant the value of which can change from line to line.

We need the following Theorems. 
Theorem $2.2([7])$. Let $1<p<\infty$ and let $\phi$ be a positive function on $\mathbb{R}_{+}$. If

$$
K_{\phi, p}=\int_{0}^{\infty} \frac{\phi(t)}{t} t^{\frac{1}{p}} d t<\infty
$$

then

$$
\left\|H_{\phi} f\right\|_{L_{p}\left(\mathbb{R}_{+}\right)} \leq K_{\phi, p}\|f\|_{L_{p}\left(\mathbb{R}_{+}\right)} .
$$

Theorem 2.3 ([17,22-24]). Let $1<p<\infty$ and let $u$ and $v$ be weight functions defined on $\mathbb{R}_{+}$. Then the inequality

$$
\left(\int_{0}^{\infty}\left|\frac{1}{x} \int_{0}^{x} f(t) d t\right|^{p} u(x) d x\right)^{\frac{1}{p}} \leq C\left(\int_{0}^{\infty}|f(x)|^{p} v(x) d x\right)^{\frac{1}{p}}
$$

holds if and only if

$$
B=\sup _{t>0}\left(\int_{t}^{\infty} \frac{u(x)}{x^{p}} d x\right)^{\frac{1}{p}}\left(\int_{0}^{t} v(x)^{1-p^{\prime}} d x\right)^{\frac{1}{p^{\prime}}}<\infty .
$$

Besides, if $C>0$ is the best constant in (2.2), then

$$
B \leq C \leq p^{\frac{1}{p}}\left(p^{\prime}\right)^{\frac{1}{p^{\prime}}} B .
$$

Theorem 2.4 ([17,22-24]). Let $1<p<\infty$ and let $u$ and $v$ be weight functions defined on $\mathbb{R}_{+}$. Then the inequality

$$
\left(\int_{0}^{\infty}\left|\int_{x}^{\infty} \frac{f(t)}{t} d t\right|^{p} u(x) d x\right)^{\frac{1}{p}} \leq C\left(\int_{0}^{\infty}|f(x)|^{p} v(x) d x\right)^{\frac{1}{p}}
$$

holds if and only if

$$
B^{\star}=\sup _{t>0}\left(\int_{0}^{t} u(x) d x\right)^{\frac{1}{p}}\left(\int_{t}^{\infty} \frac{v(x)^{1-p^{\prime}}}{x^{p^{\prime}}} d x\right)^{\frac{1}{p^{\prime}}}<\infty .
$$

Besides, if $C>0$ is the best constant in (2.4), then

$$
B^{\star} \leq C \leq p^{\frac{1}{p}}\left(p^{\prime}\right)^{\frac{1}{p^{\prime}}} B^{\star} .
$$

\section{Main results}

In this section of our paper we state and prove our principal assertions.

Theorem 3.1. Let $1<p<\infty$, and let $u$ and $v$ be increasing weight functions defined on $\mathbb{R}_{+}$. Let $\phi$ be a positive function on $\mathbb{R}_{+}$satisfying the condition

$\int_{0}^{1} \frac{\phi(t)}{t} t^{\frac{1}{p}} d t<\infty$ and there exists constants $C_{i}>0, i=1,2$ such that

$$
\frac{C_{1}}{t} \leq \phi(t) \leq \frac{C_{2}}{t} \text { for all } t \geq 1
$$

Then the inequality

$$
\left\|H_{\phi} f\right\|_{L_{p, u}\left(\mathbb{R}_{+}\right)} \leq C\|f\|_{L_{p, v}\left(\mathbb{R}_{+}\right)}
$$

holds if and only if $B<\infty$.

Besides, if $C>0$ is the best constant in (3.2), then

$$
C_{1} B \leq C \leq\left(\left(2^{\frac{1}{p^{\prime}}}+1\right)(p-1)^{\frac{1}{p}} K_{\phi, p}+2^{\frac{1}{p^{\prime}}} p^{\frac{1}{p}}\left(p^{\prime}\right)^{\frac{1}{p^{\prime}}} C_{2}\right) B .
$$


Proof. Necessity. Let $t>0$ be a fixed number. Suppose that (3.2) holds. Let $f$ be a nonnegative function defined on $(0, \infty)$ and let supp $f \subset[0, t]$. Then by $(3.1)$, we have

$$
\begin{aligned}
& \left(\int_{0}^{\infty}\left(\int_{0}^{\infty} \frac{\phi\left(\frac{x}{y}\right)}{y} f(y) d y\right)^{p} u(x) d x\right)^{\frac{1}{p}}=\left(\int_{0}^{\infty}\left(\int_{0}^{t} \frac{\phi\left(\frac{x}{y}\right)}{y} f(y) d y\right)^{p} u(x) d x\right)^{\frac{1}{p}} \\
& \geq\left(\int_{t}^{\infty}\left(\int_{0}^{t} \frac{\phi\left(\frac{x}{y}\right)}{y} f(y) d y\right)^{p} u(x) d x\right)^{\frac{1}{p}} \geq C_{1}\left(\int_{t}^{\infty} \frac{u(x)}{x^{p}} d x\right)^{\frac{1}{p}}\left(\int_{0}^{t} f(y) d y\right) .
\end{aligned}
$$

We choose the test function as $f_{t}(x)=v(x)^{1-p^{\prime}} \chi_{(0, t)}(x)$. It is obvious that

$$
\left\|f_{t}\right\|_{L_{p, v}(0, \infty)}=\left(\int_{0}^{t} v(x)^{1-p^{\prime}} d x\right)^{\frac{1}{p}}
$$

Thus, we get from (3.2)

$$
C_{1}\left(\int_{t}^{\infty} \frac{u(x)}{x^{p}} d x\right)^{\frac{1}{p}}\left(\int_{0}^{t} v(x)^{1-p^{\prime}} d y\right) \leq C\left(\int_{0}^{t} v(x)^{1-p^{\prime}} d x\right)^{\frac{1}{p}} .
$$

Therefore, one has

$$
C_{1}\left(\int_{t}^{\infty} \frac{u(x)}{x^{p}} d x\right)^{\frac{1}{p}}\left(\int_{0}^{t} v(x)^{1-p^{\prime}} d y\right)^{\frac{1}{p^{\prime}}} \leq C .
$$

Sufficiency. By (3.1), we have

$$
\begin{gathered}
\int_{0}^{\infty} \frac{\phi(t)}{t} t^{\frac{1}{p}} d t=\int_{0}^{1} \frac{\phi(t)}{t} t^{\frac{1}{p}} d t+\int_{1}^{\infty} \frac{\phi(t)}{t} t^{\frac{1}{p}} d t \\
\leq \int_{0}^{1} \frac{\phi(t)}{t} t^{\frac{1}{p}} d t+C_{2} \int_{1}^{\infty} \frac{d t}{t^{2-\frac{1}{p}}}=\int_{0}^{1} \frac{\phi(t)}{t} t^{\frac{1}{p}} d t+C_{2} p^{\prime}<\infty .
\end{gathered}
$$

Thus, condition (2.1) of Theorem 2.2 is satisfied.

Without loss of generality we may assume that the function $u$ has the form

$$
u(t)=u(0)+\int_{0}^{t} \psi(\tau) d \tau
$$

where $u(0)=\lim _{t \rightarrow+0} u(t)$ and $\psi$ is a positive function on $(0, \infty)$. In other words, let $u$ be an absolute continuous function on $(0, \infty)$. Indeed, for any increasing function $u$ on $(0, \infty)$ there exists a sequence of absolutely continuous functions $\left\{\varphi_{n}\right\}$ such that $\lim _{n \rightarrow \infty} \varphi_{n}(t)=u(t)$, $0 \leq \varphi_{n}(t) \leq u(t)$ a.e. $t>0$ and $\varphi_{n}(0)=u(0)$. Furthermore the functions $\varphi_{n}(t)$ are increasing, and besides

$$
\varphi_{n}(t)=\varphi_{n}(0)+\int_{0}^{t} \varphi_{n}^{\prime}(\tau) d \tau
$$

Hence, using Fatou's theorem, we obtain estimate (3.2) for increasing functions on $(0, \infty)$ (see [5], Theorem 4).

Let us estimate the left-hand side of inequality (3.2). We have

$$
\left(\int_{0}^{\infty}\left|H_{\phi} f(x)\right|^{p} u(x) d x\right)^{\frac{1}{p}}=\left(\int_{0}^{\infty}\left|H_{\phi} f(x)\right|^{p}\left(u(0)+\int_{0}^{x} \psi(t) d t\right) d x\right)^{\frac{1}{p}} .
$$

If $u(0)=0$, then

$$
\left(\int_{0}^{\infty}\left|H_{\phi} f(x)\right|^{p} u(x) d x\right)^{\frac{1}{p}}=\left(\int_{0}^{\infty}\left|H_{\phi} f(x)\right|^{p}\left(\int_{0}^{x} \psi(t) d t\right) d x\right)^{\frac{1}{p}} .
$$


However, if $u(0)>0$, then

Condition (2.3) implies that

$$
\begin{aligned}
& \left(\int_{0}^{\infty}\left|H_{\phi} f(x)\right|^{p} u(x) d x\right)^{\frac{1}{p}} \leq\left(\int_{0}^{\infty}\left|H_{\phi} f(x)\right|^{p} u(0) d x\right)^{\frac{1}{p}} \\
& \quad+\left(\int_{0}^{\infty}\left|H_{\phi} f(x)\right|^{p}\left(\int_{0}^{x} \psi(t) d t\right) d x\right)^{\frac{1}{p}}=E_{1}+E_{2} .
\end{aligned}
$$

$$
\begin{gathered}
\sup _{t>0}\left(\int_{t}^{\infty} \frac{u(x)}{x^{p}} d x\right)^{\frac{1}{p}}\left(\int_{0}^{t} v(x)^{1-p^{\prime}} d x\right)^{\frac{1}{p^{\prime}}} \geq \sup _{t>0}\left[\frac{u(t)}{v(t)}\right]^{\frac{1}{p}}\left(\int_{t}^{\infty} \frac{d x}{x^{p}}\right)^{\frac{1}{p}}\left(\int_{0}^{t} d x\right)^{\frac{1}{p^{\prime}}} \\
=\frac{1}{(p-1)^{\frac{1}{p}}}\left[\sup _{t>0} \frac{u(t)}{v(t)}\right]^{\frac{1}{p}} .
\end{gathered}
$$

Therefore for all $t>0$, we get

$$
u(t) \leq(p-1) B^{p} v(t) .
$$

By Theorem 2.2 and (3.3), we get

$$
\begin{gathered}
E_{1}=\left(\int_{0}^{\infty}\left|H_{\phi} f(x)\right|^{p} u(0) d x\right)^{\frac{1}{p}}=(u(0))^{\frac{1}{p}}\left(\int_{0}^{\infty}\left|H_{\phi} f(x)\right|^{p} d x\right)^{\frac{1}{p}} \\
\leq K_{\phi, p}(u(0))^{\frac{1}{p}}\left(\int_{0}^{\infty}|f(x)|^{p} d x\right)^{\frac{1}{p}} \leq K_{\phi, p}\left(\int_{0}^{\infty}|f(x)|^{p} u(x) d x\right)^{\frac{1}{p}} \\
\leq B(p-1)^{\frac{1}{p}} K_{\phi, p}\|f\|_{L_{p, v}\left(\mathbb{R}_{+}\right) .}
\end{gathered}
$$

Let us estimate the integral $E_{2}$. We have

$$
\begin{gathered}
E_{2}=\left(\int_{0}^{\infty}\left|H_{\phi} f(x)\right|^{p}\left(\int_{0}^{x} \psi(t) d t\right) d x\right)^{\frac{1}{p}}=\left(\int_{0}^{\infty}\left|H_{\phi} f(x)\right|^{p}\left(\int_{0}^{\infty} \psi(t) \chi_{\{x>t\}}(x) d t\right) d x\right)^{\frac{1}{p}} \\
=\left(\int_{0}^{\infty} \int_{0}^{\infty}\left|H_{\phi} f(x)\right|^{p} \psi(t) \chi_{\{x>t\}}(x) d t d x\right)^{\frac{1}{p}}=\left(\int_{0}^{\infty} \psi(t)\left(\int_{t}^{\infty}\left|H_{\phi} f(x)\right|^{p} d x\right) d t\right)^{\frac{1}{p}} \\
\leq 2^{\frac{1}{p}}\left(\int_{0}^{\infty} \psi(t)\left(\int_{t}^{\infty}\left|\int_{0}^{t} \frac{\phi\left(\frac{x}{y}\right)}{y} f(y) d y\right|^{p} d x\right) d t\right)^{\frac{1}{p}} \\
+2^{\frac{1}{p}}\left(\int_{0}^{\infty} \psi(t)\left(\int_{t}^{\infty}\left|\int_{t}^{\infty} \frac{\phi\left(\frac{x}{y}\right)}{y} f(y) d y\right|^{p} d x\right) d t\right)^{\frac{1}{p}}=E_{21}+E_{22} .
\end{gathered}
$$

We estimate $E_{22}$. Using Theorem 2.2 and inequality (3.3), we get

$$
\begin{aligned}
& E_{22}=2^{\frac{1}{p^{\prime}}}\left(\int_{0}^{\infty} \psi(t)\left(\int_{0}^{\infty}\left|\int_{0}^{\infty} \frac{\phi\left(\frac{x}{y}\right)}{y} f(y) \chi_{\{y>t\}}(y) d y\right|^{p} \chi_{\{x>t\}}(x) d x\right) d t\right)^{\frac{1}{p}} \\
& \leq 2^{\frac{1}{p^{\prime}}}\left(\int_{0}^{\infty} \psi(t)\left(\int_{0}^{\infty}\left|\int_{0}^{\infty} \frac{\phi\left(\frac{x}{y}\right)}{y} f(y) \chi_{\{y>t\}}(y) d y\right|^{p} d x\right) d t\right)^{\frac{1}{p}} \\
& \leq 2^{\frac{1}{p^{\prime}}} K_{\phi, p}\left(\int_{0}^{\infty} \psi(t)\left(\int_{0}^{\infty}|f(x)|^{p} \chi_{\{x>t\}}(x) d x\right) d t\right)^{\frac{1}{p}} \\
& =2^{\frac{1}{p^{\prime}}} K_{\phi, p}\left(\int_{0}^{\infty}|f(x)|^{p}\left(\int_{0}^{x} \psi(t) d t\right) d x\right)^{\frac{1}{p}} \leq 2^{\frac{1}{p^{\prime}}} K_{\phi, p}\left(\int_{0}^{\infty}|f(x)|^{p} u(x) d x\right)^{\frac{1}{p}} \\
& \leq 2^{\frac{1}{p^{\prime}}}(p-1)^{\frac{1}{p}} B K_{\phi, p}\|f\|_{L_{p, v}\left(\mathbb{R}_{+}\right)} .
\end{aligned}
$$


Now we estimate $E_{21}$. Note that if $x>t, y \leq t$, then $\frac{x}{y} \geq 1$. By virtue of condition (3.1), one has

$$
\begin{aligned}
& E_{21}=2^{\frac{1}{p^{\prime}}}\left(\int_{0}^{\infty} \psi(t)\left(\int_{t}^{\infty}\left|\int_{0}^{t} \frac{\varphi\left(\frac{x}{y}\right)}{y} f(y) d y\right|^{p} d x\right) d t\right)^{\frac{1}{p}} \\
& \leq 2^{\frac{1}{p^{\prime}}}\left(\int_{0}^{\infty} \psi(t)\left(\int_{t}^{\infty}\left(\int_{0}^{t} \frac{\varphi\left(\frac{x}{y}\right)}{y}|f(y)| d y\right)^{p} d x\right) d t\right)^{\frac{1}{p}} \\
& \leq 2^{\frac{1}{p^{\prime}}} C_{2}\left(\int_{0}^{\infty} \psi(t)\left(\int_{t}^{\infty} \frac{d x}{x^{p}}\right)\left(\int_{0}^{t}|f(y)| d y\right)^{p} d t\right)^{\frac{1}{p}} \\
& =2^{\frac{1}{p^{\prime}}}(p-1)^{-\frac{1}{p}} C_{2}\left(\int_{0}^{\infty} \psi(t) t^{1-p}\left(\int_{0}^{t}|f(y)| d y\right)^{p} d t\right)^{\frac{1}{p}} .
\end{aligned}
$$

We have

$$
\begin{gathered}
\frac{1}{p-1} \int_{t}^{\infty} \psi(s) s^{1-p} d s=\int_{t}^{\infty} \psi(s)\left(\int_{s}^{\infty} \frac{d x}{x^{p}}\right) d s \\
=\int_{0}^{\infty} \psi(s) \chi_{(t, \infty)}(s)\left(\int_{0}^{\infty} \chi_{(s, \infty)}(x) x^{-p} d x\right) d s \\
=\int_{0}^{\infty} \int_{0}^{\infty} \psi(s) x^{-p} \chi_{(t, \infty)}(s) \chi_{(s, \infty)}(x) d x d s \\
=\int_{t}^{\infty} x^{-p}\left(\int_{t}^{x} \psi(s) d s\right) d x \leq \int_{t}^{\infty} x^{-p}\left(\int_{0}^{x} \psi(s) d s\right) d x \leq \int_{t}^{\infty} \frac{u(x)}{x^{p}} d x .
\end{gathered}
$$

Therefore, we get

$$
(p-1)^{-\frac{1}{p}} \sup _{t>0}\left(\int_{t}^{\infty} \psi(s) s^{1-p} d s\right)^{\frac{1}{p}}\left(\int_{0}^{t} v(s)^{1-p^{\prime}} d s\right)^{\frac{1}{p^{\prime}}} \leq B .
$$

Thus, by Theorem 2.3 and by (3.4), we have

$$
\begin{gathered}
E_{21} \leq 2^{\frac{1}{p^{\prime}}}(p-1)^{-\frac{1}{p}} C_{2}\left(\int_{0}^{\infty} \psi(t) t^{1-p}\left(\int_{0}^{t}|f(y)| d y\right)^{p} d t\right)^{\frac{1}{p}} \\
\leq 2^{\frac{1}{p^{\prime}}} p^{\frac{1}{p}}\left(p^{\prime}\right)^{\frac{1}{p^{\prime}}} C_{2} B\left(\int_{0}^{\infty}|f(t)|^{p} v(t) d t\right)^{\frac{1}{p}}=2^{\frac{1}{p^{\prime}}} p^{\frac{1}{p}}\left(p^{\prime}\right)^{\frac{1}{p^{\prime}}} C_{2} B\|f\|_{L_{p, v}\left(\mathbb{R}_{+}\right)} .
\end{gathered}
$$

The proof is completed.

Remark 3.2. Let all the conditions of Theorem 3.1 be satisfied. Then the Hausdorff operator $H_{\phi}$ is equivalent to operator $L_{\phi}$. Here

$$
L_{\phi} f(x)=\frac{1}{x} \int_{0}^{x} f(y) d y+\int_{x}^{\infty} \frac{\phi\left(\frac{x}{y}\right)}{y} f(y) d y .
$$

In the case of decreasing weight functions the following Theorem holds.

Theorem 3.3. Let $1<p<\infty$, and let $u$ and $v$ be decreasing weight functions defined on $\mathbb{R}_{+}$. Let $\phi$ be a positive function on $\mathbb{R}_{+}$satisfying condition

$\int_{1}^{\infty} \frac{\phi(t)}{t} t^{\frac{1}{p}} d t<\infty$ and there exists constants $C_{i}^{\prime}>0, i=1,2$ such that

$$
C_{1}^{\prime} \leq \phi(t) \leq C_{2}^{\prime} \text { for all } t \leq 1 \text {. }
$$

Then the inequality

holds if and only if $B^{\star}<\infty$.

$$
\left\|H_{\phi} f\right\|_{L_{p, u}\left(\mathbb{R}_{+}\right)} \leq C\|f\|_{L_{p, v}\left(\mathbb{R}_{+}\right)}
$$


Besides, if $C>0$ is the best constant in (3.6), then

$$
C_{1}^{\prime} B^{\star} \leq C \leq\left(\left(2^{\frac{1}{p^{\prime}}}+1\right)\left(p^{\prime}-1\right)^{\frac{1}{p^{\prime}}} K_{\phi, p}+2^{\frac{1}{p^{\prime}}} p^{\frac{1}{p}}\left(p^{\prime}\right)^{\frac{1}{p^{\prime}}} C_{2}^{\prime}\right) B^{\star} .
$$

Proof. Necessity. Let $t>0$ be a fixed number. Suppose that (3.6) holds. Let $f$ be a nonnegative function defined on $(0, \infty)$ and let supp $f \subset[t, \infty)$. Then by $(3.5)$, we have

$$
\begin{aligned}
& \left(\int_{0}^{\infty}\left(\int_{0}^{\infty} \frac{\phi\left(\frac{x}{y}\right)}{y} f(y) d y\right)^{p} u(x) d x\right)^{\frac{1}{p}}=\left(\int_{0}^{\infty}\left(\int_{t}^{\infty} \frac{\phi\left(\frac{x}{y}\right)}{y} f(y) d y\right)^{p} u(x) d x\right)^{\frac{1}{p}} \\
& \geq\left(\int_{0}^{t}\left(\int_{t}^{\infty} \frac{\phi\left(\frac{x}{y}\right)}{y} f(y) d y\right)^{p} u(x) d x\right)^{\frac{1}{p}} \geq C_{1}^{\prime}\left(\int_{0}^{t} u(x) d x\right)^{\frac{1}{p}}\left(\int_{t}^{\infty} \frac{f(y)}{y} d y\right) .
\end{aligned}
$$

We choose the test function as $f_{t}(x)=(x v(x))^{1-p^{\prime}} \chi_{(t, \infty)}(x)$. It is obvious that

$$
\left\|f_{t}\right\|_{L_{p, v}(0, \infty)}=\left(\int_{t}^{\infty} \frac{v(x)^{1-p^{\prime}}}{x^{p^{\prime}}} d x\right)^{\frac{1}{p}}
$$

Thus, we get from (3.6)

$$
C_{1}^{\prime}\left(\int_{0}^{t} u(x) d x\right)^{\frac{1}{p}}\left(\int_{t}^{\infty} \frac{v(x)^{1-p^{\prime}}}{x^{p^{\prime}}} d y\right) \leq C\left(\int_{t}^{\infty} \frac{v(x)^{1-p^{\prime}}}{x^{p^{\prime}}} d x\right)^{\frac{1}{p}} .
$$

Therefore, one has

$$
C_{1}^{\prime}\left(\int_{0}^{t} u(x) d x\right)^{\frac{1}{p}}\left(\int_{t}^{\infty} \frac{v(x)^{1-p^{\prime}}}{x^{p^{\prime}}} d y\right)^{\frac{1}{p^{\prime}}} \leq C .
$$

Sufficiency. By (3.5), we have

$$
\begin{gathered}
\int_{0}^{\infty} \frac{\phi(t)}{t} t^{\frac{1}{p}} d t=\int_{0}^{1} \frac{\phi(t)}{t} t^{\frac{1}{p}} d t+\int_{1}^{\infty} \frac{\phi(t)}{t} t^{\frac{1}{p}} d t \\
\leq C_{2}^{\prime} \int_{0}^{1} t^{\frac{1}{p}-1} d t+\int_{1}^{\infty} \frac{\phi(t)}{t} t^{\frac{1}{p}}=C_{2}^{\prime} p+\int_{1}^{\infty} \frac{\phi(t)}{t} t^{\frac{1}{p}}<\infty .
\end{gathered}
$$

Thus, condition (2.1) of Theorem 2.2 is satisfied.

Without loss of generality we may assume that the function $u$ has the form

$$
u(t)=u(\infty)+\int_{t}^{\infty} \psi(\tau) d \tau,
$$

where $u(\infty)=\lim _{t \rightarrow \infty} u(t)$ and $\psi$ is a positive function on $(0, \infty)$. Indeed, for any decreasing function $u$ on $(0, \infty)$ there exists a sequence of absolutely continuous functions $\left\{\varphi_{n}\right\}$ such that $\lim _{n \rightarrow \infty} \varphi_{n}(t)=u(t), 0 \leq \varphi_{n}(t) \leq u(t)$ a.e. $t>0$ and $\varphi_{n}(\infty)=u(\infty)$. Furthermore the functions $\varphi_{n}(t)$ are decreasing, and besides

$$
\varphi_{n}(t)=\varphi_{n}(\infty)+\int_{t}^{\infty}\left(-\varphi_{n}^{\prime}(\tau)\right) d \tau
$$

Hence, using Fatou's theorem, we obtain estimate (3.6) for any decreasing functions on $(0, \infty)$ (see [5], Theorem 5).

Let us estimate the left-hand side of inequality (3.6). We have

$$
\left(\int_{0}^{\infty}\left|H_{\phi} f(x)\right|^{p} u(x) d x\right)^{\frac{1}{p}}=\left(\int_{0}^{\infty}\left|H_{\phi} f(x)\right|^{p}\left(u(\infty)+\int_{x}^{\infty} \psi(t) d t\right) d x\right)^{\frac{1}{p}} .
$$


If $u(\infty)=0$, then

$$
\left(\int_{0}^{\infty}\left|H_{\phi} f(x)\right|^{p} u(x) d x\right)^{\frac{1}{p}}=\left(\int_{0}^{\infty}\left|H_{\phi} f(x)\right|^{p}\left(\int_{x}^{\infty} \psi(t) d t\right) d x\right)^{\frac{1}{p}} .
$$

However, if $u(\infty)>0$, then

$$
\begin{gathered}
\left(\int_{0}^{\infty}\left|H_{\phi} f(x)\right|^{p} u(x) d x\right)^{\frac{1}{p}} \leq\left(\int_{0}^{\infty}\left|H_{\phi} f(x)\right|^{p} u(\infty) d x\right)^{\frac{1}{p}} \\
+\left(\int_{0}^{\infty}\left|H_{\phi} f(x)\right|^{p}\left(\int_{x}^{\infty} \psi(t) d t\right) d x\right)^{\frac{1}{p}}=F_{1}+F_{2} .
\end{gathered}
$$

Condition (2.5) implies that

$$
\begin{gathered}
\sup _{t>0}\left(\int_{0}^{t} u(x) d x\right)^{\frac{1}{p}}\left(\int_{t}^{\infty} \frac{v(x)^{1-p^{\prime}}}{x^{p^{\prime}}} d x\right)^{\frac{1}{p^{\prime}}} \geq \sup _{t>0}\left[\frac{u(t)}{v(t)}\right]^{\frac{1}{p}}\left(\int_{0}^{t} d x\right)^{\frac{1}{p}}\left(\int_{t}^{\infty} \frac{d x}{x^{p^{\prime}}}\right)^{\frac{1}{p^{\prime}}} \\
=\frac{1}{\left(p^{\prime}-1\right)^{\frac{1}{p^{\prime}}}}\left[\sup _{t>0} \frac{u(t)}{v(t)}\right]^{\frac{1}{p}} .
\end{gathered}
$$

Therefore for all $t>0$, we get

$$
u(t) \leq\left(p^{\prime}-1\right)^{p-1}\left(B^{\star}\right)^{p} v(t) .
$$

By Theorem 2.2 and (3.7), we get

$$
\begin{gathered}
F_{1}=\left(\int_{0}^{\infty}\left|H_{\phi} f(x)\right|^{p} u(\infty) d x\right)^{\frac{1}{p}}=(u(\infty))^{\frac{1}{p}}\left(\int_{0}^{\infty}\left|H_{\phi} f(x)\right|^{p} d x\right)^{\frac{1}{p}} \\
\leq K_{\phi, p}(u(\infty))^{\frac{1}{p}}\left(\int_{0}^{\infty}|f(x)|^{p} d x\right)^{\frac{1}{p}} \leq K_{\phi, p}\left(\int_{0}^{\infty}|f(x)|^{p} u(x) d x\right)^{\frac{1}{p}} \\
\leq B^{\star}\left(p^{\prime}-1\right)^{\frac{1}{p^{\prime}}} K_{\phi, p}\|f\|_{L_{p, v}\left(\mathbb{R}_{+}\right)} .
\end{gathered}
$$

Let us estimate the integral $F_{2}$. We have

$$
\begin{gathered}
F_{2}=\left(\int_{0}^{\infty}\left|H_{\phi} f(x)\right|^{p}\left(\int_{x}^{\infty} \psi(t) d t\right) d x\right)^{\frac{1}{p}}=\left(\int_{0}^{\infty}\left|H_{\phi} f(x)\right|^{p}\left(\int_{0}^{\infty} \psi(t) \chi_{\{x<t\}}(x) d t\right) d x\right)^{\frac{1}{p}} \\
=\left(\int_{0}^{\infty} \int_{0}^{\infty}\left|H_{\phi} f(x)\right|^{p} \psi(t) \chi_{\{x<t\}}(x) d t d x\right)^{\frac{1}{p}}=\left(\int_{0}^{\infty} \psi(t)\left(\int_{0}^{t}\left|H_{\phi} f(x)\right|^{p} d x\right) d t\right)^{\frac{1}{p}} \\
\leq 2^{\frac{1}{p^{\prime}}}\left(\int_{0}^{\infty} \psi(t)\left(\int_{0}^{t}\left|\int_{0}^{t} \frac{\phi\left(\frac{x}{y}\right)}{y} f(y) d y\right|^{p} d x\right) d t\right)^{\frac{1}{p}} \\
+2^{\frac{1}{p^{\prime}}}\left(\int_{0}^{\infty} \psi(t)\left(\int_{0}^{t}\left|\int_{t}^{\infty} \frac{\phi\left(\frac{x}{y}\right)}{y} f(y) d y\right|^{p} d x\right) d t\right)^{\frac{1}{p}}=F_{21}+F_{22} .
\end{gathered}
$$


We estimate $F_{21}$. Using Theorem 2.2 and inequality (3.7), we get

$$
\begin{aligned}
& F_{21}=2^{\frac{1}{p^{\prime}}}\left(\int_{0}^{\infty} \psi(t)\left(\int_{0}^{\infty}\left|\int_{0}^{\infty} \frac{\phi\left(\frac{x}{y}\right)}{y} f(y) \chi_{\{y<t\}}(y) d y\right|^{p} \chi_{\{x<t\}}(x) d x\right) d t\right)^{\frac{1}{p}} \\
& \leq 2^{\frac{1}{p^{\prime}}}\left(\int_{0}^{\infty} \psi(t)\left(\int_{0}^{\infty}\left|\int_{0}^{\infty} \frac{\phi\left(\frac{x}{y}\right)}{y} f(y) \chi_{\{y<t\}}(y) d y\right|^{p} d x\right) d t\right)^{\frac{1}{p}} \\
& \leq 2^{\frac{1}{p^{\prime}}} K_{\phi, p}\left(\int_{0}^{\infty} \psi(t)\left(\int_{0}^{\infty}|f(x)|^{p} \chi_{\{x<t\}}(x) d x\right) d t\right)^{\frac{1}{p}} \\
& =2^{\frac{1}{p^{\prime}}} K_{\phi, p}\left(\int_{0}^{\infty}|f(x)|^{p}\left(\int_{x}^{\infty} \psi(t) d t\right) d x\right)^{\frac{1}{p}} \leq 2^{\frac{1}{p^{\prime}}} K_{\phi, p}\left(\int_{0}^{\infty}|f(x)|^{p} u(x) d x\right)^{\frac{1}{p}} \\
& \leq 2^{\frac{1}{p^{\prime}}}\left(p^{\prime}-1\right)^{\frac{1}{p^{\prime}}} B^{\star} K_{\phi, p}\|f\|_{L_{p, v}\left(\mathbb{R}_{+}\right)} .
\end{aligned}
$$

Now we estimate $F_{22}$. Note that if $x<t, y \geq t$, then $\frac{x}{y} \leq 1$. By virtue of condition (3.5), one has

We have

$$
\begin{aligned}
& F_{22}=2^{\frac{1}{p^{\prime}}}\left(\int_{0}^{\infty} \psi(t)\left(\int_{0}^{t}\left|\int_{t}^{\infty} \frac{\varphi\left(\frac{x}{y}\right)}{y} f(y) d y\right|^{p} d x\right) d t\right)^{\frac{1}{p}} \\
& \leq 2^{\frac{1}{p^{\prime}}}\left(\int_{0}^{\infty} \psi(t)\left(\int_{0}^{t}\left(\int_{t}^{\infty} \frac{\varphi\left(\frac{x}{y}\right)}{y}|f(y)| d y\right)^{p} d x\right) d t\right)^{\frac{1}{p}} \\
& \leq 2^{\frac{1}{p^{\prime}}} C_{2}^{\prime}\left(\int_{0}^{\infty} \psi(t)\left(\int_{0}^{t} d x\right)\left(\int_{t}^{\infty} \frac{|f(y)|}{y} d y\right)^{p} d t\right)^{\frac{1}{p}} \\
& =2^{\frac{1}{p^{\prime}}} C_{2}^{\prime}\left(\int_{0}^{\infty} \psi(t) t\left(\int_{t}^{\infty} \frac{|f(y)|}{y} d y\right)^{p} d t\right)^{\frac{1}{p}} .
\end{aligned}
$$

$$
\begin{gathered}
\int_{0}^{t} \psi(s) s d s=\int_{0}^{t} \psi(s)\left(\int_{0}^{s} d x\right) d s=\int_{0}^{\infty} \psi(s) \chi_{(0, t)}(s)\left(\int_{0}^{\infty} \chi_{(0, s)}(x) d x\right) d s \\
=\int_{0}^{\infty} \int_{0}^{\infty} \psi(s) \chi_{(0, t)}(s) \chi_{(0, s)}(x) d x d s=\int_{0}^{t}\left(\int_{x}^{t} \psi(s) d s\right) d x \\
\leq \int_{0}^{t}\left(\int_{x}^{\infty} \psi(s) d s\right) d x \leq \int_{0}^{t} u(x) d x
\end{gathered}
$$

Therefore, we get

$$
\sup _{t>0}\left(\int_{0}^{t} \psi(s) s d s\right)^{\frac{1}{p}}\left(\int_{t}^{\infty} \frac{v(s)^{1-p^{\prime}}}{s^{p^{\prime}}} d s\right)^{\frac{1}{p^{\prime}}} \leq B^{\star} .
$$

Thus, by Theorem 2.4 and by (3.8), we have

$$
\begin{gathered}
F_{22} \leq 2^{\frac{1}{p^{\prime}}} C_{2}^{\prime}\left(\int_{0}^{\infty} \psi(t) t\left(\int_{t}^{\infty} \frac{|f(y)|}{y} d y\right)^{p} d t\right)^{\frac{1}{p}} \\
\leq 2^{\frac{1}{p^{\prime}}} p^{\frac{1}{p}}\left(p^{\prime}\right)^{\frac{1}{p^{\prime}}} C_{2}^{\prime} B^{\star}\left(\int_{0}^{\infty}|f(t)|^{p} v(t) d t\right)^{\frac{1}{p}}=2^{\frac{1}{p^{\prime}}} p^{\frac{1}{p}}\left(p^{\prime}\right)^{\frac{1}{p^{\prime}}} C_{2}^{\prime} B^{\star}\|f\|_{L_{p, v}\left(\mathbb{R}_{+}\right)} .
\end{gathered}
$$

This completes the proof. 
Remark 3.4. Let all the conditions of Theorem 3.3 be satisfied. Then the Hausdorff operator $H_{\phi}$ is equivalent to the operator $M_{\phi}$. Here

$$
M_{\phi} f(x)=\int_{0}^{x} \frac{\phi\left(\frac{x}{y}\right)}{y} f(y) d y+\int_{x}^{\infty} \frac{f(y)}{y} d y .
$$

Remark 3.5. Let $u$ and $v$ be general weight functions defined on $\mathbb{R}_{+}$. We note that Hardy type inequalities for the Hausdorff operator is proved in [19]. In [19] the obtained necessary conditions differ from the sufficient conditions as well as that both depend not only on weights but also on the kernel that generate the Hausdorff operator. For the Hardy and its adjoint operators, the obtained necessary and sufficient conditions coincide and reduce to the classical ones.

Remark 3.6. Let $u$ and $v$ be general weight functions defined on $\mathbb{R}_{+}$. We note that two-weight inequalities for the Hausdorff operator is also proved in [4]. In [4] the obtained necessary conditions also differs from the sufficient conditions. For multidimensional Hausdorff operator similar inequalities is proved in [6].

\subsection{Examples for environments}

We summarize the paper with some examples.

Example 3.7. Let $1<p<\infty$ and let $\alpha>-\frac{1}{p}$. Suppose that $u$ and $v$ are increasing weight functions defined on $\mathbb{R}_{+}$. Suppose that

$$
\phi(x)=\left\{\begin{array}{lll}
x^{\alpha}, & \text { if } & 0<x<1, \\
\left|\sin \frac{1}{x}\right|, & \text { if } & x \geq 1 .
\end{array}\right.
$$

Then, we get the operator

$$
H_{\phi} f(x)=\int_{0}^{x} \frac{\left|\sin \frac{y}{x}\right|}{y} f(y) d y+x^{\alpha} \int_{x}^{\infty} \frac{f(y)}{y^{\alpha+1}} d y .
$$

It is obvious that $\phi$ satisfy conditions of Theorem 3.1 with $C_{1}=\frac{2}{\pi}$ and $C_{2}=1$. Also, $K_{\phi, p}=\frac{p}{\alpha p+1}+\int_{1}^{\infty} x^{\frac{1}{p}-1}\left|\sin \frac{1}{x}\right| d x$.

Then the inequality (3.2) holds if and only if $B<\infty$. Besides, if $C>0$ is the best constant in $(3.2)$, then

$$
\frac{2}{\pi} B \leq C \leq\left(\left(2^{\frac{1}{p^{\prime}}}+1\right)(p-1)^{\frac{1}{p}} K_{\phi, p}+2^{\frac{1}{p^{\prime}}} p^{\frac{1}{p}}\left(p^{\prime}\right)^{\frac{1}{p^{\prime}}}\right) B .
$$

Example 3.8. Let $1<p<\infty$ and let $\beta>0$. Suppose that $u$ and $v$ are increasing weight functions defined on $\mathbb{R}_{+}$. Suppose that

$$
\phi(t)=\left\{\begin{array}{llc}
(1-x)^{\beta-1}, & \text { if } & 0<x<1, \\
\log \left(1+\frac{1}{x}\right), & \text { if } & x \geq 1 .
\end{array}\right.
$$

Then, we get the operator

$$
H_{\phi} f(x)=\int_{x}^{\infty} \frac{(y-x)^{\beta-1}}{y^{\beta}} f(y) d y+\int_{0}^{x} \frac{\log \left(1+\frac{y}{x}\right)}{y} f(y) d y .
$$

It is easy to see that $\phi$ satisfy conditions of Theorem 3.1 with $C_{1}=\frac{1}{2}$ and $C_{2}=1$. Also, $K_{\phi, p}=B\left(\frac{1}{p}, \beta\right)+\int_{1}^{\infty} x^{\frac{1}{p}-1} \log \left(1+\frac{1}{x}\right) d x$ and $B$ is the Euler beta function. 
Then the inequality (3.2) holds if and only if $B<\infty$. Besides, if $C>0$ is the best constant in $(3.2)$, then

$$
\frac{B}{2} \leq C \leq\left(\left(2^{\frac{1}{p^{\prime}}}+1\right)(p-1)^{\frac{1}{p}} K_{\phi, p}+2^{\frac{1}{p^{\prime}}} p^{\frac{1}{p}}\left(p^{\prime}\right)^{\frac{1}{p^{\prime}}}\right) B .
$$

Example 3.9. Let $1<p<\infty$ and let $\phi(x)=e^{-x}$. Suppose that $u$ and $v$ are decreasing weight functions defined on $\mathbb{R}_{+}$.

Then, we get the operator

$$
H_{\phi} f(x)=\int_{0}^{\infty} \frac{e^{-\frac{x}{y}}}{y} f(y) d y
$$

It is obvious that $\phi$ satisfies conditions of Theorem 3.3 with $C_{1}^{\prime}=\frac{1}{e}$ and $C_{2}^{\prime}=1$. Also, $K_{\phi, p}=\Gamma\left(\frac{1}{p}\right)$ and $\Gamma$ is the Gamma function.

Then the inequality (3.6) holds if and only if $B^{\star}<\infty$. Besides, if $C>0$ is the best constant in (3.6), then

$$
\frac{1}{e} B^{\star} \leq C \leq\left(\left(2^{\frac{1}{p^{\prime}}}+1\right)(p-1)^{\frac{1}{p}} \Gamma\left(\frac{1}{p}\right)+2^{\frac{1}{p^{\prime}}} p^{\frac{1}{p}}\left(p^{\prime}\right)^{\frac{1}{p^{\prime}}}\right) B^{\star} .
$$

Example 3.10. Let $1<p<\infty$. Suppose that $u$ and $v$ are decreasing weight functions defined on $\mathbb{R}_{+}$. Assume that

$$
\phi(x)=\left\{\begin{array}{llc}
\frac{1}{x+1}, & \text { if } & 0<x<1 \\
\frac{x^{1-\frac{1}{p}}}{1+x^{2}}, & \text { if } & x \geq 1
\end{array}\right.
$$

Then, we get the operator

$$
H_{\phi} f(x)=x^{1-\frac{1}{p}} \int_{0}^{x} \frac{y^{\frac{1}{p}}}{x^{2}+y^{2}} f(y) d y+\int_{x}^{\infty} \frac{f(y)}{x+y} d y .
$$

It is easy to see that $\phi$ satisfies conditions of Theorem 3.3 with $C_{1}^{\prime}=\frac{1}{2}$ and $C_{2}^{\prime}=1$. Also, $K_{\phi, p}=\frac{\pi}{4}+\int_{0}^{1} \frac{x^{\frac{1}{p}-1}}{x+1} d x$.

Then the inequality (3.6) holds if and only if $B^{\star}<\infty$. Besides, if $C>0$ is the best constant in $(3.2)$, then

$$
\frac{B^{\star}}{2} \leq C \leq\left(\left(2^{\frac{1}{p^{\prime}}}+1\right)(p-1)^{\frac{1}{p}} K_{\phi, p}+2^{\frac{1}{p^{\prime}}} p^{\frac{1}{p}}\left(p^{\prime}\right)^{\frac{1}{p^{\prime}}}\right) B^{\star} .
$$

Let us give some examples of weight functions satisfying conditions of Theorem 3.1 and Theorem 3.3, respectively.

Example 3.11. Let $1<p<\infty$. Suppose $u(x)=x^{\alpha}$ and let $v(x)=e^{x}$.

Then the inequality (3.2) holds if and only if $0<\alpha<p-1$.

Example 3.12. Let $1<p<\infty$ and let $0<\alpha \leq \beta<p-1$. Suppose $u(x)=\log ^{\alpha}(1+x)$ and let $v(x)=(1+x)^{\beta}$.

Then the inequality (3.2) holds.

Example 3.13. Let $1<p<\infty$ and let $u(x)=v(x)=x^{\alpha}$.

Then the inequality (3.6) holds if and only if $-1<\alpha<0$.

Example 3.14. Let $1<p \leq 2$. Suppose $u(x)=\frac{1}{1+x}$ and let $v(x)=\frac{\left(1+x^{2}\right)^{p-1}}{x^{p}}$.

Then the inequality (3.6) holds. 
Acknowledgment. The research of first author was partially supported by the Peoples' Friendship University of Russia and by the grant of 1st Azerbaijan-Russia Joint Grant Competition Grant N EIF-BGM-4-RFTF-1/2017-21/01/1-M-08. We would like to thank both reviewers for their valuable comments and suggestions on the manuscript.

\section{References}

[1] D.R. Aliyeva, On the boundedness of Hardy type integral operators in weighted Lebesgue space, Caspian J. Appl. Math., Ecol. Econ. 6 (1), 35-41, 2018.

[2] K.F. Andersen, Boundedness of Hausdorff operators on $L_{p}\left(\mathbb{R}^{n}\right), H^{1}\left(\mathbb{R}^{n}\right)$, and $B M O\left(\mathbb{R}^{n}\right)$, Acta Sci. Math. (Szeged) 69 (1-2), 409-418, 2003.

[3] R.A. Bandaliev, On one inequalities for convolution type operator, Hacet. J. Math. Stat. 42 (3), 199-210, 2013.

[4] R.A. Bandaliyev and P. Górka, Hausdorff operator in Lebesgue spaces, Math. Inequal. Appl. 22 (2), 657-676, 2019.

[5] R.A. Bandaliev and K.K. Omarova, Two-weight norm inequalities for certain singular integrals, Taiwanese J. Math. 16 (2), 713-732, 2012.

[6] R.A. Bandaliyev and K.H. Safarova, On boundedness of multidimensional Hausdorff operator in weighted Lebesgue spaces, Tbilisi Math. J. 13 (1), 39-45, 2020.

[7] G. Brown and F. Mòricz, The Hausdorff operator and the quasi Hausdorff operators on the space $L^{p}, 1<p<\infty$, Math. Inequal. Appl. 3 (1), 105-115, 2000.

[8] G. Brown and F. Mòricz, Multivariate Hausdorff operators on the spaces $L^{p}\left(\mathbb{R}^{n}\right)$, J. Math. Anal. Appl. 271 (2), 443-454, 2002.

[9] J. Chen, D. Fan and J. Li, Hausdorff operators on function spaces, Chin. Ann. Math. Ser. B, 33 (4), 537-556, 2013.

[10] J. Chen and X. Wu, Best constant for Hausdorff operators on n-dimensional product spaces, Sci. China Math. 57 (3), 569-578, 2014.

[11] D.E. Edmunds, V. Kokilashvili and A. Meskhi, Bounded and Compact Integral Operators, Kluwer Academic Publishers, 2002.

[12] G. Gao, X. Wu and W. Guo, Some results for Hausdorff operators, Math. Inequal. Appl. 18 (1), 155-168, 2015.

[13] A. Gogatishvili and V.D. Stepanov, Reduction theorems for weighted integral inequalities on the cone of monotone functions, Russian Math. Surveys 68 (4), 597-664, 2013.

[14] V.S. Guliyev, Two-weighted inequalities for integral operators in $L_{p}$-spaces and their applications, Proc. Steklov Math. Inst. 204 (3), 97-116, 1993.

[15] A. Hussain and A. Ajaib, Some weighted inequalities for Hausdorff operators and commutators, J. Inequal. Appl. 2018, Article No. 6, 2018.

[16] A. Hussain and G. Gao, Multidimensional Hausdorff operators and commutators on Herz-type spaces, J. Inequal. Appl. 2013, Article No. 594, 2013.

[17] A. Kufner, L. Maligranda and L.E. Persson, The Hardy inequality: About its history and some related results, Research report, Department of Mathematics, Luleå University of Technology, Sweden, 2005.

[18] S.N Lal and S. Ram, On the absolute Hausdorff summability of a Fourier series, Pacific J. Math. 42 (2), 439-451, 1972.

[19] E. Liflyand, Hardy type inequalities in the category of Hausdorff operators, Modern Meth. Oper. Theory Harmonic Anal. OTHA 2018. Springer Proc. Math. \& Stat., 291, 81-91, 2019.

[20] E. Liflyand and A. Miyachi, Boundedness of the Hausdorff operators in $H^{p}$ spaces, $0<p<1$, Studia Math. 194 (3), 279-292, 2009.

[21] E. Liflyand and A. Miyachi, Boundedness of multidimensional Hausdorff operators in $H^{p}$ spaces, $0<p<1$, Trans. Amer. Math. Soc. 371 (7), 4793-4814, 2019. 
[22] V.G. Maz'ya, Sobolev Spaces, Springer-Verlag, Berlin, 1985.

[23] G. Talenti, Osservazione sopra una classe di disuguaglianze, Rend. Sem. Mat. Fiz. Milano 39 (1), 171-185, 1969.

[24] G. Tomaselli, A class of inequalities, Boll. Unione Mat. Ital. 2 (1), 622-631, 1969. 